\title{
Cerebral Air Embolism After Gastrointestinal Procedure: A Case Report and Literature Review
}

\author{
Iyad Farouji ${ }^{a}$, Kok Hoe Chan ${ }^{\mathrm{a}, \mathrm{f}}$, Hossam Abed ${ }^{\mathrm{a}}$, Theodore DaCosta ${ }^{\mathrm{a}}$, Baris Vefali ${ }^{\mathrm{b}}$, \\ Ormena Joseph ${ }^{\mathrm{a}}$, Jihad Slima, c, Theodore DaCosta, Jra, d, Addi Suleiman ${ }^{\mathrm{a}, \mathrm{e}}$
}

\begin{abstract}
Esophagogastroduodenoscopy (EGD) is one of the forefronts of minimally invasive modalities with excellent safety records and tremendous capability but despite its accolades and functions, there are still very rare complications including air embolism. It is a life-threatening condition that could lead to a significant increase in morbidity and mortality. However, there are limited data for incidence of air embolism in association with gastrointestinal endoscopy. Diagnosis of air embolism after or during gastrointestinal endoscopy might be a difficult task due to overlapping presentations with anesthesia effects on the cardiopulmonary and the neurological systems, as a result, there should be increased awareness allowing clinicians to quickly rule out air embolism in patient with altered mental status or cardiopulmonary changes after or during gastrointestinal endoscopy. Herein, we report a unique case of cerebral air embolism after EGD in a 79-year-old female patient. In addition, we also performed a systematic review of cases based on PRISMA guideline, with the aim to investigate the demographics and clinical outcomes associated with this complication. This systematic review of cases hopes to increase the awareness about this rare entity.
\end{abstract}

Keywords: Cerebral air embolism; Gas embolism; Endoscopy; Endoscopic retrograde cholangiopancreatography; Literature review

\section{Introduction}

The entry of air into the circulation, which is usually iatrogenic,

Manuscript submitted December 22, 2020, accepted January 4, 2021

Published online January 19, 2021

aDepartment of Medical Education, Saint Michael's Medical Centre, New York Medical College, Newark, NJ, USA

bSaint George's University School of Medicine, West Indies

'Department of Infectious Disease, Saint Michael's Medical Centre, New York Medical College, Newark, NJ, USA

${ }^{\mathrm{d} D e p a r t m e n t}$ of Gastroenterology, Saint Michael's Medical Centre, New York Medical College, Newark, NJ, USA

eDepartment of Cardiology, Saint Michael's Medical Centre, New York Medical College, Newark, NJ, USA

${ }^{f}$ Corresponding Author: Kok Hoe Chan, Saint Michael's Medical Center, Newark, NJ 07101, USA. Email: kchan2@primehealthcare.com

doi: https://doi.org/10.14740/jmc3639 is a serious condition that can cause life-threatening complications such as ischemic stroke with a high morbidity and mortality [1]. After entering the venous system, it can travel to the cerebral circulation by different ways, including cardiac shunts, retrograde cerebral venous embolism, or through arterial-venous malformations [2]. Endoscopy-related cerebral air embolism is a rare complication that can occur with different procedures such as endoscopic retrograde cholangiopancreatography (ERCP), while being relatively rare in esophagogastroduodenoscopy (EGD), endoscopic ultrasonography (EUS), colonoscopy, or sigmoidoscopy [3]. The estimated incidence of cerebral air embolism post-ERCP is around $0.0033 \%$, dropping to $0.00056 \%$ in post-EGD [4]. Several factors may increase the risk of air embolism after these procedures including anatomic anomalies, malignancies, previous interventions or surgeries of the bile duct system, inflammation of the digestive system, particular interventional techniques and others [5]. In the systemic review conducted by Donepudi et al, systemic air embolism, including pulmonary, venous, arterial and cerebral circulation have been reported as a potential complication of gastrointestinal (GI) endoscopy in general [5]. Nonetheless, there were no previous studies or review of literature focused exclusively on the cerebral air embolism after upper endoscopy, its clinical presentations, risk factors and clinical outcomes. Herein, we present a very unique case of a 79-year-old female patient, who had recurrent cerebral air embolism with ischemic stroke after upper endoscopy. In addition, we also performed a systematic review of cases based on PRISMA guideline, with the aim to investigate the demographics and clinical outcomes associated with this complication (Supplementary Material 1, www.journalmc.org).

\section{Case Report}

A 79-year-old female patient with a past medical history of hypertension, previous stroke and Schatzki rings treated with dilation 7 years ago presented with progressive dysphagia to solid food. The patient reported the sensation of food getting stuck in her throat with associated coughing and choking spells. Seven years ago, she had similar symptoms and was treated with endoscopy and esophageal balloon dilation, which was complicated by a stroke resulting in left-sided weakness. No clear cause of the stroke was found at that time. Eventually her weakness improved with physical and occupational therapy.

During this admission, a conventional video-endoscopy 

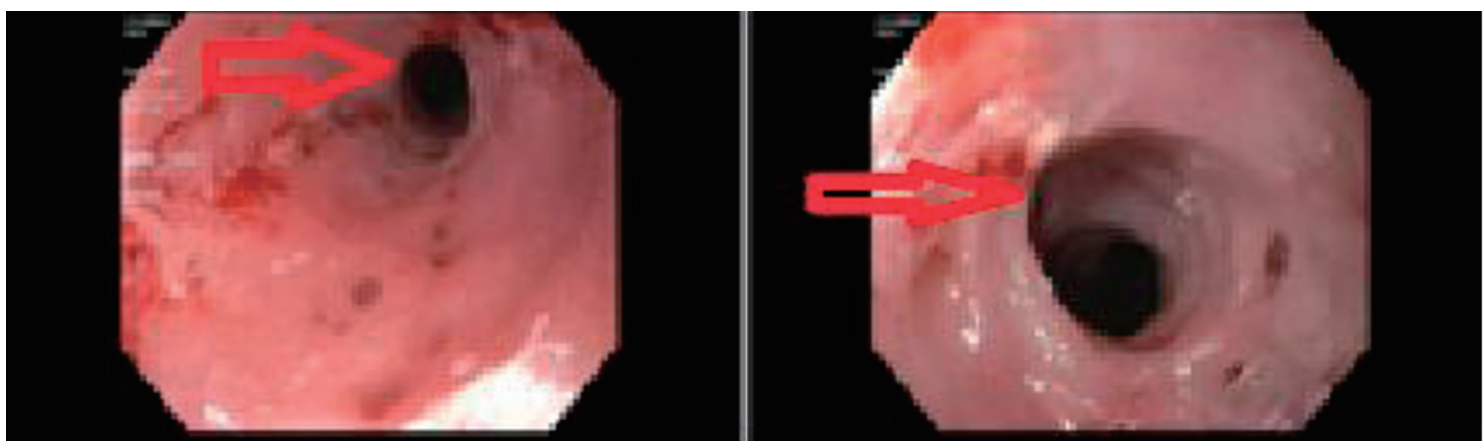

Figure 1. Endoscopy which showed esophageal ring (red arrows) in the proximal esophagus, $15 \mathrm{~cm}$ from the incisors.

was performed with propofol injection for sedation throughout the procedure. At the onset of the procedure, the patient had a brief episode of hypoxia with a drop in her oxygen saturation to $70 \%$. The scope was removed, and she was ventilated with Ambu bag resulting in an increase in oxygenation to $100 \%$. The procedure was then resumed. The patient was monitored during the procedure with pulse oximetry, blood pressure monitoring and continuous telemetry electrocardiogram (ECG) tracing. Endoscopy showed an esophageal ring in the proximal esophagus, $15 \mathrm{~cm}$ from the incisors (Fig. 1). The ring was traversed with moderate resistance. Further down the esophagus, many linear esophageal ulcers with oozing blood and small diverticula were appreciated. A through the scope (TTS) dilator was passed through the scope and a dilation with a $10 \mathrm{~mm}$ balloon was performed at the site of the ring. The dilation showed mild improvement with a small mucosal tear. The scope was able to be passed to the stomach and duodenum which were found to be normal mucosa with unremarkable findings.

After the procedure, the patient failed to regain conscious and was intubated for airway protection and kept on mechanical ventilation in the intensive care unit. An urgent head computed

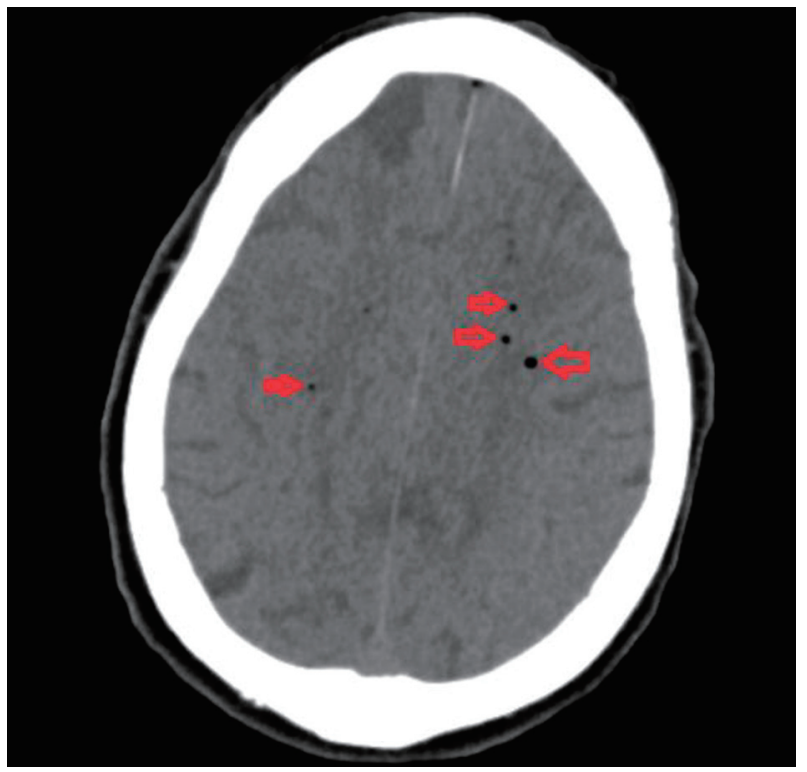

Figure 2. Computed tomography of head showing multiple foci of intraparenchymal air (red arrows) suggestive of air emboli. tomography (CT) showed multiple foci of intraparenchymal air suggestive of air emboli as well as visualization of small infarcts in the left frontal lobe (Fig. 2). Transthoracic echocardiogram was performed, which showed no evidence of patent foramen ovale or atrial septal defect on bubble study. The patient's condition worsened over the next $12 \mathrm{~h}$. She became hypotensive, her hypoxia worsened, and brainstem reflexes were absent. Repeat head CT showed diffuse cerebral edema and mass effect along with obliteration of the basilar cisterns as well as uncal, transtentorial and developing tonsillar herniation. CT angiogram of the head and neck showed occlusion of the intracranial carotid arteries, mid and distal cervical internal carotid arteries, anterior and middle cerebral arteries, and distal posterior cerebral artery branches (Fig. 3). Although mechanical ventilation and other

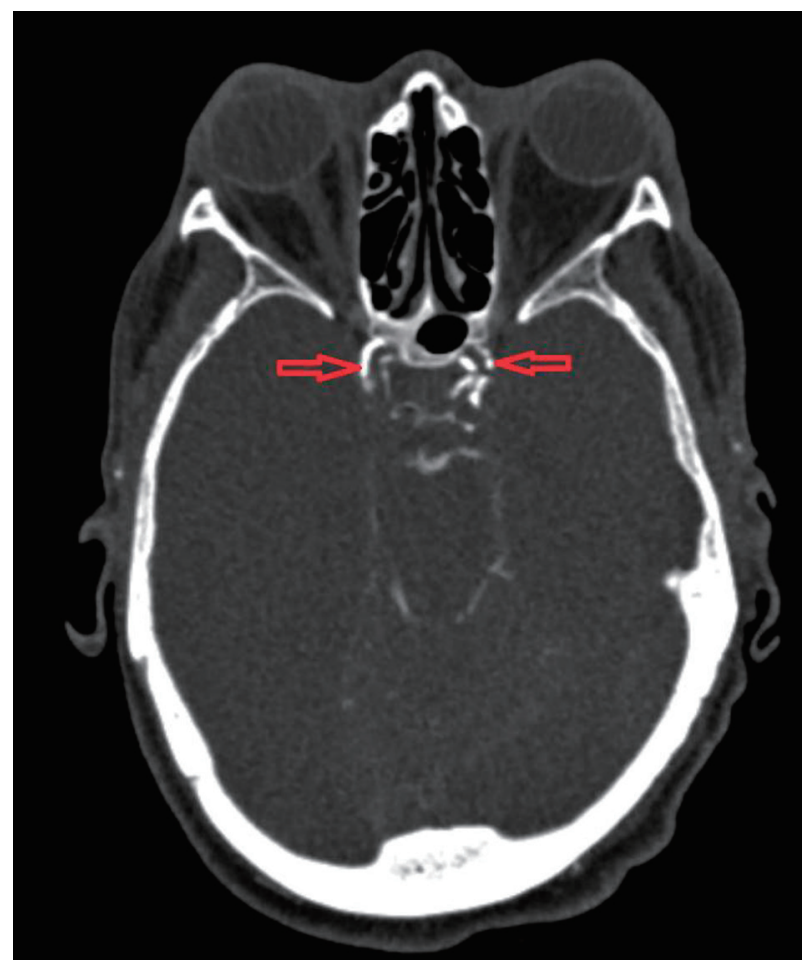

Figure 3. Computed tomography angiogram of the head and neck showing occlusion of the intracranial carotid arteries (red arrows), mid and distal cervical internal carotid arteries, anterior and middle cerebral arteries, and distal posterior cerebral artery branches. 


\section{PRISMA Flow Diagram}

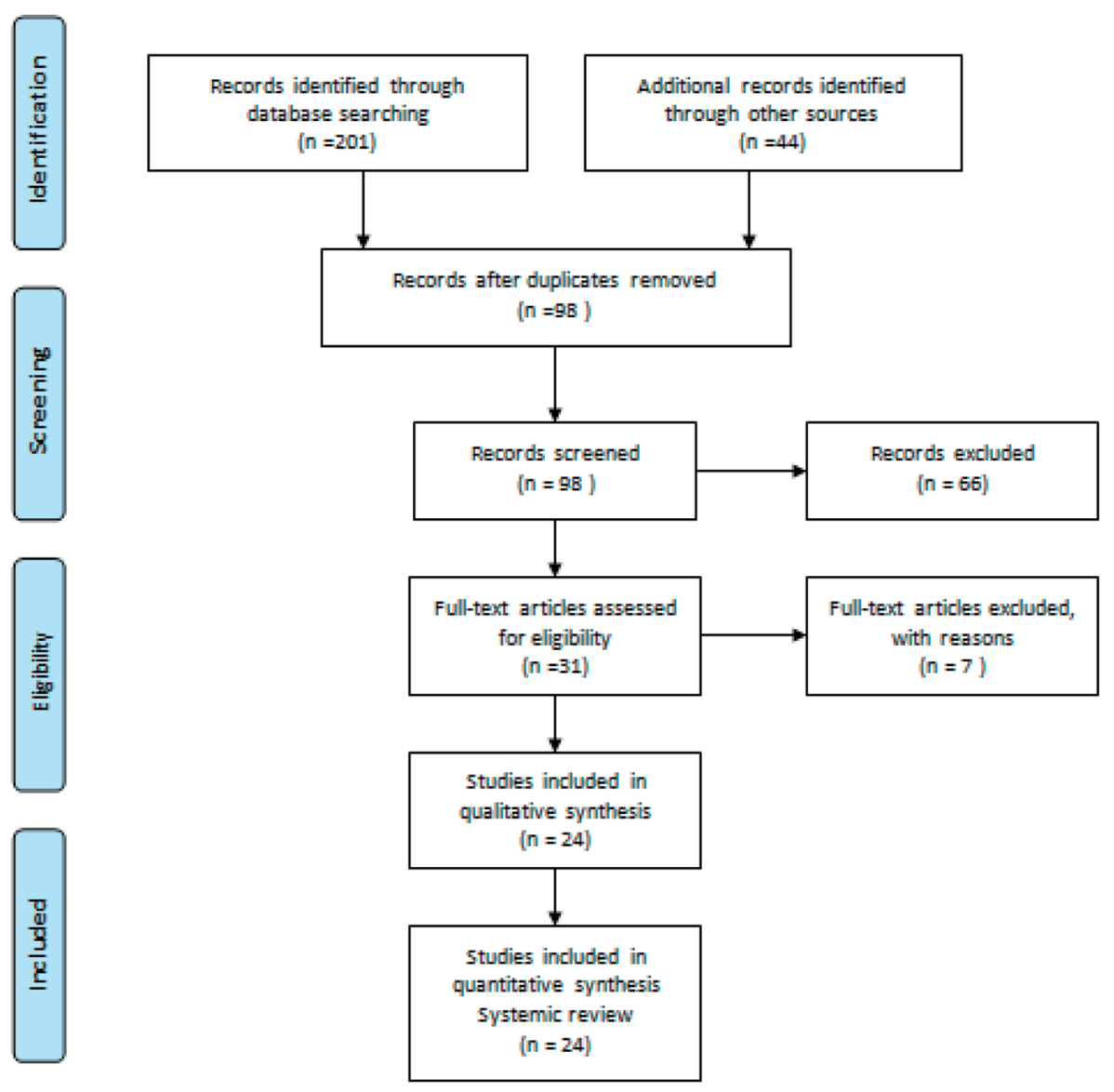

Figure 4. PRISMA flow chart of study selection.

supportive measures were continued, the patient passed away a few hours later in the intensive care unit.

\section{Methodology}

A systematic review of the literature was performed using the Preferred Reporting Items for Systematic Reviews and MetaAnalyses (PRISMA) guidelines, in order to identify all studies of patients with cerebral air embolism. Literature search was conducted in databases such as PubMed/MEDLINE, Google Scholar, Ovid and Web of Science from 1998 to 2020. Search item included the keywords of "air embolism AND (EGD or ERCP)", "cerebral air embolism AND (EGD or ERCP)", "gas embolism AND (EGD or ERCP)" and "cerebral gas embolism AND (EGD or ERCP)". All study designs included case reports and case series were eligible for final analysis. We excluded guidelines that 1) were not published in English, and 2) were published before the year 1998 .

Two reviewers independently assessed the titles and abstracts retrieved from the search. Full text papers of relevant titles and abstracts were then obtained, which were then assessed for eligibility based on the inclusion criteria. Any discrepancies were resolved through discussion to reach a consensus in the presence of the third and fourth reviewers. A PRISMA flow chart of study selection is shown in Figure 4.

For each eligible study, demographic data were extracted including number of patients, age, sex and clinical characteristics (medical history, surgical history, social history, medications, presenting symptoms and type of procedures used). We also included the CT, echocardiogram and magnetic resonance imaging (MRI) findings. Statistical analysis was performed with GraphPad Prism statistical software. The data were tabulated, and outcomes were cumulatively analyzed. Continuous variables were expressed as mean \pm standard deviation, while categorical variables were expressed as frequencies or percentages.

\section{Results}

Twenty-four studies were included in the final analysis, with 
Table 1. Demographic Characteristics, Clinical History, Types of Procedure and Endoscopic Findings of Reviewed Cases

\begin{tabular}{|c|c|}
\hline $\mathrm{N}$ & 26 \\
\hline Average age & 64.7 \\
\hline \multicolumn{2}{|l|}{ Gender } \\
\hline Male & 16 \\
\hline Female & 10 \\
\hline \multicolumn{2}{|l|}{ Past medical history } \\
\hline History of malignancy & 11 \\
\hline Unknown & 10 \\
\hline \multicolumn{2}{|l|}{ Types of procedure } \\
\hline EGD & 13 \\
\hline ERCP & 13 \\
\hline \multicolumn{2}{|l|}{ Use of sedation } \\
\hline Yes & 15 \\
\hline No & 2 \\
\hline Unknown & 9 \\
\hline \multicolumn{2}{|l|}{ Type of gas used in the procedure } \\
\hline Air & 2 \\
\hline $\mathrm{CO}_{2}$ & 0 \\
\hline Unknown & 24 \\
\hline \multicolumn{2}{|l|}{ Endoscopy findings } \\
\hline Stricture & 14 \\
\hline Diverticulum & 1 \\
\hline Varices & 2 \\
\hline Esophageal bleeding & 1 \\
\hline Esophagitis/gastritis & 3 \\
\hline Duodenal AV malformation & 1 \\
\hline Calculus in the extrahepatic biliary tree & 1 \\
\hline Unknown & 4 \\
\hline
\end{tabular}

EGD: esophagogastroduodenoscopy; ERCP: endoscopic retrograde cholangiopancreatography; AV: arteriovenous.

a total of 26 patients. Demographic characteristics of patients are presented in the table below. The average age was 64.7 years old (ranged 42 to 87$)$. There were $16(61.5 \%)$ male and $10(38.4 \%)$ female.

Clinical characteristics are presented in the table below. Surprisingly $11(42.3 \%)$ of the patients had underlying malignancy. As for types of procedure performed, 13 of them were EGD and 14 were ERCP. Use of sedation, including conscious and general anesthesia, was observed in $15(57.7 \%)$ of patients. As for the endoscopic findings, presence of strictures requiring stenting or dilatation seemed to be the highest risk for air embolism. This was observed in close to $14(53.8 \%)$ of patients. The detailed descriptions are outlined in Table 1.

As for imaging findings, presence of right to left shunt and patent foramen ovale were observed in nine $(34.6 \%)$ patients; only six $(22.2 \%)$ patients had normal echocardiogram and no evidence of right to left shunt. On the other hand, 11 (40.7\%) patients had no documented echocardiogram findings. The detailed imaging findings are outlined in Table 2.

All patients presented with signs of hypoxia with low oxygen saturation, bradycardia and loss of consciousness (100\%). Ten patients survived but majority had extremities paralysis with only two that returned to normal baseline. There were about $14(53.8 \%)$ of patients expired and two had no documented outcome. The detailed clinical signs and symptoms are outlined in Table 3.

\section{Discussion}

The undesired entrance of air into the vascular system can cause gas embolism and can occur in either the venous or arterial system depending on its entrance point into the systemic circulation [6]. Gas embolism is a life-threatening condition and can lead to disastrous conditions and significant morbidity and mortality [7]. Different gases can lead to this condition including air, which is the most common cause, carbon dioxide, nitrous oxide, nitrogen and others [8]. Most of the times, this situation is iatrogenic and can occur during procedures such as EGD, colonoscopy, enteroscopy, sigmoidoscopy, endoscopic ultrasound, ERCP, surgical procedures, intravenous catheterization, hemodialysis, interventional radiological procedures, positive pressure ventilation and trauma [9]. In this literature review, we will focus on cerebral gas embolism after different gastroenterology procedures.

There are some conditions, situations and procedures that put patients at higher risk for gas embolism. We can categorize these conditions into GI tract inflammation, anatomical anomalies, decreased blood supply, malignancy and procedures. GI tract inflammation breaks down into infections, abscesses and inflammatory bowel disease [10]. Anatomical anomalies are commonly caused by strictures, arterio-venous malformations and biliary atresia [11]. Decreased blood supply can be seen in necrotizing enterocolitis and mesenteric ischemia [2]. Furthermore, GI malignancies seem to be a major risk factor of gas embolization [12]. After reviewing the literature, 11 (42.3\%) of the cases that we analyzed were found to have GI tumors. Previous interventions and surgical procedures of the GI tract also carry this risk and may arise from the procedure itself or from complications of the procedure [13]. The risk of air embolization from GI procedures can be based on technique. The rate, pressure and amount of gas insufflation during endoscopy, use of different gases and the procedure itself can increase the risk. Procedures such as ERCP and interventions such as stenting, biopsies and sphincterotomies all have an increased risk of air embolization due to the increased manipulation of the intestinal mucosa [14-16].

Different theories on the mechanism of gas entry into the vascular system during GI procedures have been described in literature. This entrance breaks down into two parts. The entry of gas from the GI tract into the portal venous system and the translocation of the gas bubbles from the portal venous system into the systemic circulation allow it to settle in the cerebral circulation. Many hypotheses for the first step include 
Table 2. Imaging Findings of the Reviewed Cases

\begin{tabular}{ll}
\hline N & \\
CT findings & 26 \\
$\quad$ Positive findings of air in brain parenchymal or pneumocephalus or presence of air in the cerebral artery & 23 \\
$\quad$ Unknown & 3 \\
Echocardiogram findings & 9 \\
$\quad \begin{array}{l}\text { Presence of right to left shunt } \\
\text { No right to left shunt }\end{array}$ & 6 \\
$\quad$ Unknown & 11 \\
\hline
\end{tabular}

CT: computed tomography.

intramural dissection of insufflated air into the portal vein, air entry through exposed vessels in a gastric or esophageal ulcer, transection of duodenal vein radicles, transgression of air into adjacent veins from inflammation of the mucosa, and entry into the venous system through biliary-venous fistulas or shunts and vascular cannulation with adjacent vessels from GI tumors [17-19].

After settling in the venous system, the gas bubbles can migrate into the central nervous system. Some mechanisms allowing for this migration include the paradoxical embolization through existing right to left heart shunts from a preexisting anomaly. Patent foramen ovale has been reported in around nine $(34.6 \%)$ of the cases that we included in our study. Moreover, retrograde flow via the superior vena cava into the cerebral veins and direct return of gas bubbles to the left atrium through the pulmonary veins after failure in filtering the air emboli into the pulmonary circulation are other

Table 3. Clinical Signs and Symptoms of Reviewed Cases

\begin{tabular}{ll}
\hline $\mathrm{N}$ & 26 \\
Hypoxia with low $\mathrm{SpO}_{2}$ & \\
Yes & 26 \\
No & 0 \\
Heart rate & \\
$\quad$ Bradycardia & 26 \\
Tachycardia & 0 \\
Normal & 0 \\
Mentation & \\
Loss of consciousness & 26 \\
Awake & 0 \\
Confused & 0 \\
Outcome & \\
Alive & 10 \\
$\quad$ Extremities paralysis & 7 \\
Spatial disability & 1 \\
Normal & 2 \\
\hline Expired & 14 \\
Unknown & 2 \\
\hline
\end{tabular}

possible mechanisms $[11,16,17]$. Tommasino et al described another theory of causing cerebral air embolism without the presence of any shunts if the air embolism is large enough in size [20].

The diagnosis of cerebral air embolisms can be very challenging during the procedure, especially in sedated patients. More than half of the patients who we reviewed were sedated during the procedure, which may play an important role in delaying the diagnosis of the stroke, or the sedation itself may increase the risk of air embolism. It is important to have a high clinical suspicion in patients who show any signs of complication during the procedure. For neurological signs and symptoms during the procedure, as all the patients who have been reported presented with bradycardia, hypoxia and altered mental status, physicians and clinical staff should continue to monitor the heart rate, the oxygen saturation and the mental status. Other symptoms and signs include headache, seizures, blindness, aphasia, failure to regain consciousness after the procedure, neurological deficits, hemiparesis, quadriparesis or paraplegia conjugate eye deviation, pupillary dilation and bilateral extensor plantar reflex $[3,21]$.

Patients who present with any signs or symptoms of air emboli should have an urgent CT scan of the head without contrast looking for intracranial gas either in the vessels or parenchyma and assess for any midline shift, cerebral edema, loss of differentiation between the white and gray matter, and parenchymal herniation [22]. MRI can further show areas of acute infarcts. Due to rapid reabsorption of air from the brain arterioles, immediate imaging should be performed to prevent missing the diagnosis in these patients [23]. After diagnosis, all patients should have an echocardiogram to assess if the patient has an intracardiac shunt, patent foramen ovale, or defect that may be the cause of air transfer [24]. Of note, transesophageal echocardiogram revealed higher sensitivity and specificity in comparison to transthoracic when identifying intracardiac shunts [25].

In cases where air embolism is on the differential during a procedure, the procedure should be terminated immediately, and the patient should be stabilized [26]. The stomach and duodenum should be decompressed to prevent further gas migration to the vasculature, and it is preferred to place the patient in a Trendelenburg position to prevent the gas bubbles from translocating to the brain [26]. All patients should receive $100 \%$ oxygen, even if they are not hypoxic, to generate 
a diffusion gradient preventing further bubble formation [27]. Another recommendation is early hyperbaric oxygen therapy. Hyperbaric oxygen therapy may reduce air bubble size, accelerate nitrogen reabsorption and increase the oxygen content of arterial blood, which may reduce ischemia [28, 29].

Finally, we suggest the utilization of prophylactic measures, especially in patients who are at high risk of cerebral air embolism as complications from GI procedures. We recommend the use of carbon dioxide during the procedure instead of air because it is easily and rapidly absorbed in comparison to air [30]. We also suggest repairing intracardiac shunts in high-risk patients before undergoing GI procedures and interventions. Herein, we present a rare case of cerebral air embolism after endoscopy with esophageal dilation. What makes our case more unique is the patient had a recurrent stroke after a similar procedure and the absence of an intracardiac shunt.

\section{Conclusion}

Cerebral air embolism during endoscopy is a rare complication. There are different hypotheses that may explain the mechanism of formation and translocation of the air embolus from the GI tract to the central nervous system. History of malignancy and presence of strictures requiring stenting or dilatation through endoscopy seems to be the highest risk for air embolism. The aim of this case report and literature review is to increase awareness about this entity among endoscopists. In addition, we encourage conducting larger studies to quantify the risk of post-endoscopic air embolism.

\section{Supplementary Material}

Suppl 1. A Systematic Review of Cases Based on PRISMA Guideline.

\section{Acknowledgments}

None to declare.

\section{Financial Disclosure}

None to declare.

\section{Conflict of Interest}

All authors declare no conflict of interest.

\section{Informed Consent}

Healthcare proxy has given the written informed consent to publish the case including publication of images.

\section{Author Contributions}

Iyad Farouji, Kok Hoe Chan and Hossom Abed contributed to the conception and design, acquisition, analysis and interpretation of data, as well as participate in drafting and revision of the manuscript. Theodore DaCosta, Baris Vefali and Ormena Joseph actively participate in analysis of data, drafting and revision of manuscript. Jihad Slim, Theodore DaCosta, Jr. and Addi Suleiman contributed to idea design, data analysis and critically revised the manuscript and as well as approved the final submission of manuscript.

\section{Data Availability}

The authors declare that data supporting the findings of this study are available within the article.

\section{References}

1. Lanke G, Adler DG. Gas embolism during endoscopic retrograde cholangiopancreatography: diagnosis and management. Ann Gastroenterol. 2019;32(2):156-167.

2. Kennedy C, Larvin M, Linsell J. Fatal hepatic air embolism following ERCP. Gastrointest Endosc. 1997;45(2):187188.

3. Trabanco S, Pardo S, Williams M, Diaz J, Ruiz C. Cerebral air embolism after ERCP. J Clin Anesth. 2017;36:133135.

4. Olaiya B, Adler DG. Air embolism secondary to endoscopy in hospitalized patients: results from the National Inpatient Sample (1998-2013). Ann Gastroenterol. 2019;32(5):476-481.

5. Donepudi S, Chavalitdhamrong D, Pu L, Draganov PV. Air embolism complicating gastrointestinal endoscopy: A systematic review. World J Gastrointest Endosc. 2013;5(8):359-365.

6. McAree BJ, Gilliland R, Campbell DM, Lucas JW, Dickey W. Cerebral air embolism complicating esophagogastroduodenoscopy (EGD). Endoscopy. 2008;40(Suppl 2):E191-192.

7. Gordy S, Rowell S. Vascular air embolism. Int J Crit Illn Inj Sci. 2013;3(1):73-76.

8. McCarthy CJ, Behravesh S, Naidu SG, Oklu R. Air Embolism: Diagnosis, Clinical Management and Outcomes. Diagnostics (Basel). 2017;7(1):5.

9. Casoni D, Mirra A, Goepfert C, Petruccione I, Spadavecchia C. Iatrogenic cerebral arterial gas embolism from flushing of the arterial line in two calves. Acta Vet Scand. 2018;60(1):51.

10. Christl SU, Scheppach W, Peters U, Kirchner T. Cerebral air embolism after gastroduodenoscopy: complication of a duodenocaval fistula. Gastrointest Endosc. 1994;40(3):376-378.

11. Sasaki T, Hasegawa T, Kimura T, Okada A, Mushiake S, Matsushita T. Development of intrapulmonary arteriovenous shunting in postoperative biliary atresia: evalu- 
ation by contrast-enhanced echocardiography. J Pediatr Surg. 2000;35(11):1647-1650.

12. Lamparter S, Goecke W, Koehler HH. Hepatic portal venous gas after upper endoscopy in a patient with a gastrointestinal stromal tumor. J Clin Ultrasound. 2009;37(7):401-402.

13. Maccarone G, Shakoor T, Ellis B. Air embolism after percutaneous transhepatic biliary drainage and subsequent endoscopic retrograde cholangiopancreatography (ERCP). Endoscopy. 2011;43(Suppl 2 UCTN):E399.

14. Efthymiou M, Raftopoulos S, Antonio Chirinos J, May GR. Air embolism complicated by left hemiparesis after direct cholangioscopy with an intraductal balloon anchoring system. Gastrointest Endosc. 2012;75(1):221-223.

15. Lowdon JD, Tidmore TL, Jr. Fatal air embolism after gastrointestinal endoscopy. Anesthesiology. 1988;69(4):622623.

16. Katzgraber F, Glenewinkel F, Fischler S, Rittner C. Mechanism of fatal air embolism after gastrointestinal endoscopy. Int J Legal Med. 1998;111(3):154-156.

17. Romberg C. Systemic air embolism after ERCP: a case report and review of the literature (with video). Gastrointest Endosc. 2009;70(5):1043-1045.

18. Bisceglia M, Simeone A, Forlano R, Andriulli A, Pilotto A. Fatal systemic venous air embolism during endoscopic retrograde cholangiopancreatography. Adv Anat Pathol. 2009;16(4):255-262.

19. Ben-Zvi JS, Siegel JH, Yatto R. Opacification of the portal system during ERCP: demonstration of an anomalous pancreatico-portal connection in a patient with pancreatic carcinoma. Gastrointest Endosc. 1989;35(5):445-447.

20. Tommasino C, Rizzardi R, Beretta L, Venturino M, Piccoli S. Cerebral ischemia after venous air embolism in the absence of intracardiac defects. J Neurosurg Anesthesiol. 1996;8(1):30-34.
21. Vachalova I, Ernst S, Vynogradova I, Wohrmann S, Heckmann JG. Cerebral air embolism via port catheter and endoscopic retrograde cholangio-pancreatography. Springerplus. 2013;2:477.

22. Mishra R, Reddy P, Khaja M. Fatal cerebral air embolism: a case series and literature review. Case Rep Crit Care. 2016;2016:3425321.

23. Caulfield AF, Lansberg MG, Marks MP, Albers GW, Wijman CA. MRI characteristics of cerebral air embolism from a venous source. Neurology. 2006;66(6):945-946.

24. Shahjehan RD, Abraham J. Intracardiac Shunts. In: StatPearls. Treasure Island (FL): StatPearls Publishing; 2020.

25. Thanigaraj S, Valika A, Zajarias A, Lasala JM, Perez JE. Comparison of transthoracic versus transesophageal echocardiography for detection of right-to-left atrial shunting using agitated saline contrast. Am J Cardiol. 2005;96(7):1007-1010.

26. Durant TM, Long J, Oppenheimer MJ. Pulmonary (venous) air embolism. Am Heart J. 1947;33(3):269-281.

27. Annane D, Troche G, Delisle F, Devauchelle P, Paraire F, Raphael JC, Gajdos P. Effects of mechanical ventilation with normobaric oxygen therapy on the rate of air removal from cerebral arteries. Crit Care Med. 1994;22(5):851857.

28. Blanc P, Boussuges A, Henriette K, Sainty JM, Deleflie M. Iatrogenic cerebral air embolism: importance of an early hyperbaric oxygenation. Intensive Care Med. 2002;28(5):559-563.

29. Muth CM, Shank ES. Gas embolism. N Engl J Med. 2000;342(7):476-482.

30. Wang WL, Wu ZH, Sun Q, Wei JF, Chen XF, Zhou DK, Zhou L, et al. Meta-analysis: the use of carbon dioxide insufflation vs. room air insufflation for gastrointestinal endoscopy. Aliment Pharmacol Ther. 2012;35(10):11451154. 